\title{
Adaptive TTCM Aided Near-Instantaneously Adaptive Dynamic Network Coding for Cooperative Cognitive Radio Networks
}

\author{
Wei Liang, Hung Viet Nguyen, Soon Xin Ng and Lajos Hanzo \\ School of ECS, University of Southampton, SO17 1BJ, UK. \\ Tel: +44-23-8059 3125, Fax: +44-23-8059 4508 \\ Email: \{wl7g09,hvn08r,sxn,lh\}@ecs.soton.ac.uk, http://www-mobile.ecs.soton.ac.uk
}

\begin{abstract}
An Adaptive Dynamic Network Coding (ADNC) scheme conceived for Cooperative Cognitive Radio (CCR) is proposed for devising a novel ADNC-CCR system. The system is designed for supporting communications between multiple Primary Users (PUs) and a common Base Station (BS), where the independent source information is transmitted from the PUs to the BS with the aid of multiple Cognitive Users (CUs) acting as Relay Nodes (RNs). In order to facilitate the recovery of the source information at the BS, the CUs invoke the ADNC technique, which is assisted by our cooperative protocol operating by exchanging the CCR-based control information between near-instantaneously Adaptive Turbo Trellis Coded Modulation (ATTCM) and network coding codec as well as between the CUs and the BS. More particularly, nearinstantaneously ATTCM is employed for appropriately adjusting both the modulation mode as well as the code rate of the channel coding itself and of the network coding, according to the nearinstantaneous channel conditions. As a result, our novel ADNCCCR system constructed on the basic of our holistic approach is capable of providing an increased throughput, despite reducing the transmission-period of the PU. This reduced transmissionperiod can also be directly translated into an increased duration for secondary communications of the CUs. In our proposed ADNC-CCR scheme, both the PUs and CUs employ our ATTCM scheme. As an additional novelty, the network encoder may also be activated in its adaptive mode for supporting the CUs, depending on the Boolean value of the feedback flags generated based on the success/failure of the ATTCM decoder and of the network decoder, which is evaluated and fed back by the BS. Quantitatively, it was found that the joint holistic design of our ATTCM-ADNC-CCR scheme is either capable of freeing up an approximately $40 \%$ of the PU's bandwidth in comparison to its non-cooperative counterpart, or increasing the attainable throughput by 2 bit/symbol.
\end{abstract}

Index Terms-Cognitive Radio network, Cooperative Communication, Dynamic Network Coding, ATTCM.

\section{INTRODUCTION}

The popular Cognitive Radio (CR) mechanism is capable of exploiting the temporarily available spectrum holes in the frequency domain. If a spectral-slot is not used by the Primary Users (PUs), then the Cognitive Users (CUs) (or secondary users) would gain an opportunity to access it for their secondary communications based on the $\mathrm{CR}$ technique

The financial support of the European Research Council's Advanced Fellow Grant, that of the India-UK Advanced Technology Centre, as well as that of the European Union's Seventh Framework Program (FP7/2007-2013) under the auspices of the CONERTO project (grant agreement no 288502) is gratefully acknowledged.
[1]-[3]. Spectrum allocation and sharing techniques have been widely investigated, in order to exploit the spectral bands more efficiently and to provide mutual benefits for the PUs and CUs [4]. However, both the availability and quality of a released spectral band is crucially influenced by both the specific activity and the competition between the PUs and CUs [4]. In this context, the most common paradigms in spectrum allocation and sharing are the underlay, overlay and interwave schemes [5]. In the underlay scheme, the CUs are allowed to transmit their data simultaneously with PUs, provided that the interference imposed remains below the tolerate limit. In contrast to the underlay scheme, the interference imposed on the PUs under the overlay scheme may be offset by using part of the CU's power to relay the PU's information. Finally, in the interwave scheme, the CUs avoid simultaneous transmissions with the PUs [5].

Cooperative communication constitutes a novel paradigm that promises significant improvements by providing either an improved integrity or an increased throughput with the advent of user cooperation [6], [7]. Hence, in recent years, numerous exciting new applications of relay aided communications have emerged [8]. More specifically, we have investigated the applications of relay aided communications which may involve not only the Physical Layer [9], but also the Medium Access Control [10], and the network layer as well as their cross-layer operation [11]. One of the emerging applications is based on supporting communications between the source and destination nodes with the aid of cooperative protocols. A sophisticated medium access protocol was designed in [10], [12] for communications between the source and relay nodes, which relied on efficient modulation and coding [13], [14]. Moreover, in network-coding aided systems designed for multi-user operation, the users can also act as cooperating partners or relays in order to share their resources and to assist each other in their information transmission [15]-[18]. The performance of CR schemes relying on one-way relaying was documented in [19] and two-way relaying in [20]. We found that $40 \%$ bandwidth reduction of PUs can be achieved by user cooperation.

Network Coding (NC), which was proposed by Ahlswede, Cai, Li and Yeung in 2000 [21], is capable of beneficially increasing the network capacity by invoking coding at the nodes of a network, in contrast to simply supporting routing 
functions. According to Yeung's paper [22]: "NC has propagated to various fields in engineering in the past decade, including wireless communications, channel coding, computer networks, switching theory, cryptography, computer networks, data storage and computer science." Moreover, NC has been shown to be capable of increasing the achievable throughput, robustness and security, while minimising both the amount of dissipated energy as well as the delay of packets travelling through the network [21], [23]. The benefits of NC aided cooperative systems were studied in [24]-[26] with the most important one being the reduction of redundant information transmissions [26].

Dynamic Network Codes (DNC) were proposed by Ming in [15], where each user broadcasts its own information frames both to the Base Station (BS) as well as to the other users during the first transmission period. After this phase, each user transmits a non-binary linear combination of its own frame as well as of the other users' information frames, to the BS [15]. The family of Generalized Dynamic Network Codes (GDNC) [16], [17] constitutes an extension of DNCs. In contrast to [15], in the proposal of [17] each user is allowed to broadcast several information frames during the broadcast phase via orthogonal channels [17], as well as to transmit several non-binary linear combinations, as parity frames during the cooperative phase via orthogonal channels. In order to increase the average transmission rate of GDNC without reducing its diversity order, in [16] an adaptive network code design has been proposed. The design of network codes for multi-user, multi-relay scenarios has been investigated in [18], [27], where the users transmit their independent information to the BS with the aid of the RNs. In line with the system model proposed in [18], we assume that the CUs act as the RNs to help the PUs in transmitting their information to the BS. More specifically, in our proposed scheme, the CUs are capable of relaying the PU's message, while superimposing their own messages at the same time. This leads both to an increased overall throughput and to the reduction of the required transmission period of both the PUs and the CUs, thereby creating additional time-slots (TSs) for supporting additional users.

In this paper, we consider cooperation between the PUs and CUs, where the CUs may act as network-coding aided relay nodes (RNs) for the sake of conveying information transmitted from the PUs. Our contributions in [19], [20] only considered simple RNs without the capability of invoking network coding. More explicitly, the network coding schemes of [15]-[18] were intrinsically amalgamated with an active cooperation based overlay network conceived for a CR system. In contrast to [28], we considered intelligent CUs that are capable of employing network coding techniques for their data transmission. Additionally, we invoked Adaptive Dynamic Network Coding (ADNC) [16], where the CUs deliver their information to the BS, only if the BS failed to recover the source message from the PUs. Additionally, our proposed ADNC aided cooperative cognitive radio (CCR) scheme is intrinsically amalgamated with near-instantaneously adaptive coded modulation. Explicitly, we have opted for using bandwidth-efficient Turbo Trellis Coded Modulation
(TTCM) [29], [30], because it was shown to outperform Trellis Coded Modulation (TCM), Bit-Interleaved Coded Modulation (BICM) and iteratively-decoded BICM [29].

Based on this background, the novel contribution of this paper is that a realistic Adaptive TTCM (ATTCM) arrangement is designed for the sake of conceiving an ADNC-CCR system in order to simultaneously improve both the system's throughput and its resilience. More specifically:

1) An active cooperation aided DNC scheme was proposed for overlay based CCR schemes, in order to lease the PU's bandwidth to the group of CUs for their secondary communication.

2) A bandwidth-efficient ATTCM arrangement is conceived for our proposed scheme for achieving a substantial performance improvement. The transmission rate/throughput of the system is adapted according to the near-instantaneous channel conditions, where a higher-throughput but vulnerable TTCM scheme is employed, when the channel conditions are good, while a lower-throughput but robust TTCM scheme or no transmission is used, when the near-instantaneous channel conditions are poor. Specifically, our ATTCM scheme is designed by considering the effects of both quasi-static Rayleigh fading as well as of uncorrelated Rayleigh fading.

3) Moreover, as prophesied in [16], we have conceived an adaptive DNC technique for our novel CCR system, where the CUs adaptively deliver their parity frames to the BS, depending on the success/failure of the PU's transmissions. Our proposed ADNC-CCR scheme relies on a cutting-edge channel coding scheme, which increases the achievable multiplexing gain, despite imposing a low complexity.

4) In our proposed system, each transmission link employs different ATTCM modes. During each broadcast session, all PUs transmit using the same fixed TTCM mode, which corresponds to that determined by the specific direct link having the lowest quality. Then in the cooperative phase, the "best" $C U$ would support all the transmissions towards the BS with the aid of its highest-throughput ATTCM mode. The received SNRs at the CUs and the BS may be used for determining the maximum throughput of each transmission link.

5) Finally, we conceived a novel ATTCM aided ADNC scheme for overlay CCR systems, which is capable of simultaneously exploiting the advantages of ATTCM and ADNC for facilitating a further improvement of our system's performance.

The paper is organized as follows. Section II presents our system model and outlines the network coding scheme, the ATTCM scheme as well as the ADNC. Our novel ATTCMADNC-CCR arrangement is described in Section III. The performance of our proposed scheme is evaluated in Section IV. Finally, our conclusions are presented in Section V.

\section{System MODEL}

In our proposed system portrayed in Fig. 1, we consider the uplink (UL) transmission of the ADNC-CCR network. ATTCM is advocated for judiciously selecting a suitable modulation mode according to the near-instantaneous channel condition experienced in each transmission link, which would lead to the reduction of the PU's transmission power and/or to the increase of the overall system throughput, hence 


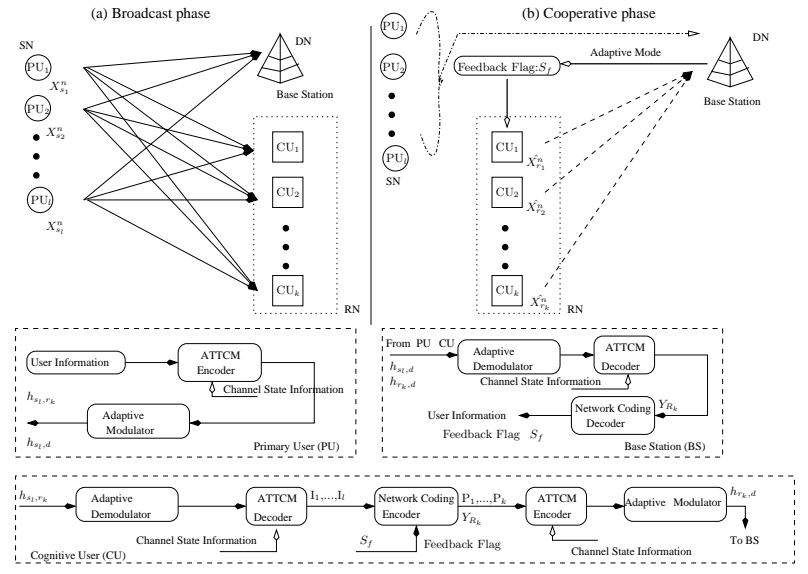

Fig. 1. The architecture of the ATTCM aided DNC in proposed CCR scheme.

simultaneously saving bandwidth for the CUs. We assume that each PU has a direct transmission link to the BS, as seen in Fig. 1. Additionally, we consider $L$ PUs, where each PU broadcasts its information to a single BS and to $K$ CUs during the broadcast phase. The BS then decodes the source information received from the PUs. Accordingly, the CUs encode the received information frames for constructing the corresponding parity frames, which are then transmitted to the BS during the cooperative phase. Hence the BS receive the information frames from the PUs and also the parity frames from the CUs. We denote the network coded codewords transmitted by the $k$ th CU/RN as $Y_{R k}$. The specific calculation of $Y_{R k}$ will be detailed in Section II-B.

During the "(a) broadcast phase" seen in Fig. 1, the $l$ th PU/SN broadcasts its information frame $X_{s_{l}}^{n}$ within the $n$th frame to both the CUs/RNs and to the BS. The signal received at the BS via the Source-to-Destination (SD) link is given by:

$$
y_{s_{l} d}^{n}=\sqrt{G_{s_{l} d}} \sqrt{P_{S}} h_{s_{l} d} X_{s_{l}}^{n}+n,
$$

while that received at the $k \mathrm{CU} / \mathrm{RN}$ via the Source-to-Relay (SR) link is:

$$
y_{s_{l} r_{k}}^{n}=\sqrt{G_{s_{l} r_{k}}} \sqrt{P_{S}} h_{s_{l} r_{k}} X_{s_{l}}^{n}+n,
$$

where $P_{S}$ is the transmission power per unit frequency emanating from the PU/SN and $n \in N$, where $N$ is the total number of frames. The $(N \times L)$ PUs simultaneously broadcast $(N \times L)$ information frames during the time duration $T_{1}$. Since a network-coding aided DAF protocol is employed at each CU/RN, the $k$ th RNs forward the decoded and reencoded information frame $\hat{X_{r_{k}}^{n}}$ during the $n$th frame to the BS. Then, during the "(b) cooperative phase" of Fig. 1, the signal received at the BS via the relay-to-destination (RD) link can be formulated as:

$$
y_{r_{k} d}^{n}=\sqrt{G_{r_{k} d}} \sqrt{P_{C U}} h_{r_{k} d} \hat{X_{r_{k}}^{n}}+n,
$$

where the CU/RN forwards the source information frame using the power $P_{C U}$. Similar to the broadcast phase, the overall transmissions during the cooperative phase are within the time duration of $T_{2}$. In our proposed system, the CUs act as the RNs, where the RNs are located half way between the PUs and BS. Accordingly, the Reduced-Distance-Related-PathlossReduction (RDRPR) [31], [32] experienced by the SR link is given by:

$$
G_{s r}=\left(\frac{d_{s d}}{d_{s r}}\right)^{\alpha},
$$

where $\alpha$ is the path-loss exponent [33]. Similarly, the RDRPR of the RD link with respect to the SD link is given by:

$$
G_{r d}=\left(\frac{d_{s d}}{d_{r d}}\right)^{\alpha} .
$$

Naturally, the RDRPR of the SD link with respect to itself is unity, i.e. we have $G_{s_{l} d}=1$. We consider an outdoor environment [33], where $\alpha=3$. Note that the same RDRPR is exploited by all the SR and RD links in our system. Thus, we have $G_{s_{l} r_{k}}=G_{r_{k} d}=2^{3}=8$. Moreover, we consider a single non-dispersive transmission path for the SD, SR and RD links of our novel ATTCM-ADNC-CCR scheme. Each of the channels in Eq. (1), Eq. (2) and Eq. (3) is comprised of two components, which may be expressed as:

$$
h=h_{s} \cdot h_{f},
$$

where the slow fading (or quasi static fading ) coefficient $h_{s}$ is constant for all symbols within a transmit frame. By contrast, the fast-fading (small-scale Rayleigh fading) coefficient $h_{f}$ varies on a symbol by symbol basis, which will described in Section II-A. According to Eq. (1), Eq. (2) and Eq. (3), the average received SNR at node $b$ per frame is given by:

$$
\begin{aligned}
\gamma_{R} & =\frac{G_{a b} E\left[|x|^{2}\right] E\left[\left|h_{f}^{2}\right|\right] E\left[\left|h_{s}^{2}\right|\right]}{N_{0}}, \\
& =\frac{G_{a b}\left|h_{a b}\right|^{2}}{N_{0}}
\end{aligned}
$$

where we have $E\left[|x|^{2}\right]=1$ and $E\left[\left|h_{f}^{2}\right|\right]=1$ for uncorrelated Rayleigh fading channels, which varies on a symbol by symbol basis, as discussed in Section II-A and we have $E\left[\left|h_{s}^{2}\right|\right]=$ $\left|h_{s}\right|^{2}=h_{a b}^{2}$. Then we determine the received SNRs of the $[l(k+1)+k]$ th communication links by referring to Fig. 1, which are $\left[\gamma_{s_{l} d}, \gamma_{s_{l} r_{k}}, \gamma_{r_{k} d}\right]$.

\section{A. Adaptive TTCM}

Employing ATTCM has the advantage that the system's effective throughput can be increased upon increasing the code rate and constellation size, when the channel-quality improves which is achieved without any bandwidth expansion. Furthermore, the bit error ratio (BER) and FER performance of the system may be improved [34]. In Fig. 1, both the PUs and CUs have employed ATTCM encoders, where the TTCM encoder comprises a pair of identical parallel-concatenated TCM encoders [35] linked by a symbol interleaver. The first TCM encoder directly processes the original input bit sequence, while the second one encodes the interleaved or scrambled version of the input bit sequence. Then the bitto-symbol mapper maps the input bits to complex-valued ATTCM symbols using the classic Set Partition based labelling method [34]. Additionally, the BS decodes the information delivered from the PUs and CUs by the ATTCM decoder. The structure of the TTCM decoder is similar to that of binary turbo codes, where each decoder alternately processes its corresponding encoder's channel-impaired output symbol, 
and then the other encoder's channel-impaired output symbol [34, pg.764]. As shown in Fig. 1, we invoked a nearinstantaneously adaptive TTCM scheme for protecting the SR and the RD links, where the effective throughput range is given by $R_{\text {tran }}=\{0,1,2,3,4,5\}$ BPS when no transmission, QPSK, 8PSK, 16QAM, 32QAM and 64QAM are considered, respectively.

Moreover, the ATTCM mode switching thresholds $\Upsilon$ $=\left[\gamma_{0}, \gamma_{1}, \gamma_{2}, \gamma_{3}, \gamma_{4}\right]$ were determined based on the FER performance curves of each of the five TTCM schemes when communicating over a Rayleigh fading channel, which is shown in Fig. 2. Specifically, both the ATTCM mode switching

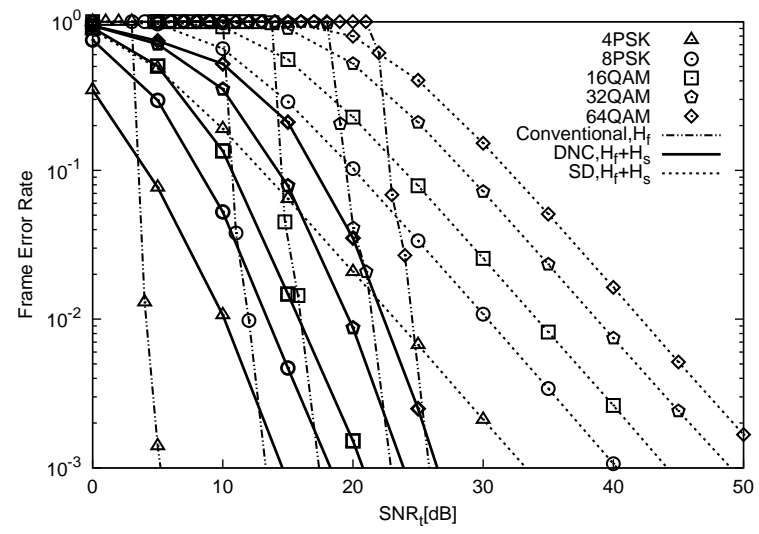

Fig. 2. The FER performance versus $\mathrm{SNR}_{t}$ of TTCM for five fixed modulation modes. "Conventional" represents the FER performance of TTCM using a frame length of 12,000 symbols, when communicating over Rayleigh fading channels. Four TTCM iterations were invoked. "DNC" and "SD" denote the FER performance of TTCM-DNC and of the TTCM aided noncooperative scheme for five fixed modulation modes using $10^{6}$ frames, when communicating over the combined uncorrelated Rayleigh fading and quasistatic fading channels.

operation and the transmission rate of the modes are based on the following algorithm:

$$
\text { MODE }= \begin{cases}\gamma_{4} \leq \gamma_{R}, & \text { TTCM-64QAM, } C^{*}=5 \mathrm{iBPS} ; \\ \gamma_{3} \leq \gamma_{R}<\gamma_{4}, & \text { TTCM-32QAM, } C^{*}=4 \mathrm{iBPS} ; \\ \gamma_{2} \leq \gamma_{R}<\gamma_{3}, & \text { TTCM-16QAM, } C^{*}=3 \mathrm{iBPS} ; \\ \gamma_{1} \leq \gamma_{R}<\gamma_{2}, & \text { TTCM-8PSK, } C^{*}=2 \mathrm{iBPS} ; \\ \gamma_{0} \leq \gamma_{R}<\gamma_{1}, & \text { TTCM-4PSK, } C^{*}=1 \mathrm{iBPS} ;\end{cases}
$$

where $C^{*}$ is the effective throughput in terms of the number of information bit per symbol (iBPS). Furthermore, we have considered two cases, when we have $\gamma_{R}<\gamma_{0}$ for the ATTCM mode switching operation. In the first case (case 1 ), no transmission is invoked in order to save energy when $\gamma_{R}<\gamma_{0}$. By contrast, the second case (case 2) invokes the 4PSK modulation mode when $\gamma_{R}<\gamma_{0}$. Thus the output $C^{*}$ of these two cases may be expressed as:

$$
C^{*}\left(\gamma_{R}<\gamma_{0}\right)= \begin{cases}0, & \text { case } 1: \text { No transmission; } \\ 1, & \text { case } 2: \text { TTCM-4PSK; }\end{cases}
$$

As seen from Fig. 2, we chose the switching thresholds carefully to ensure that the FER at the RN is lower than $10^{-3}$, in order to minimize the potential error propagation from the CUs to the BS, which are given by $\Upsilon_{A T T C M}=[5.22,12.25$, $16.10,21.15,24.49] \mathrm{dB}$.

\section{B. Network Encoder and Decoder}

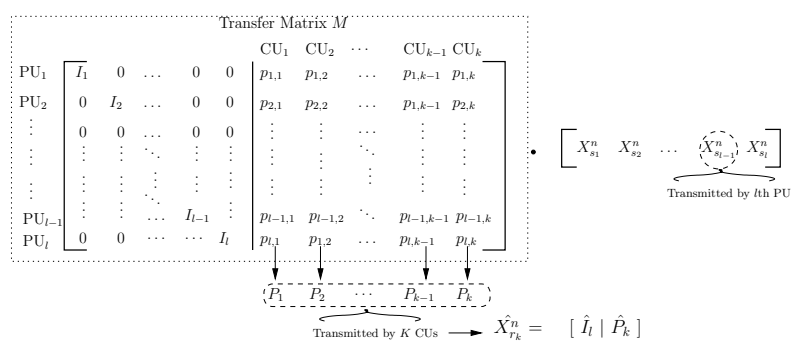

Fig. 3. Illustration of the transfer matrix and the network encoding process engaged in the DNC-CCR scheme.

This section is devoted to detailing the encoding/decoding process of the network coding technique. Fig. 1 shows how the CUs would successfully deliver the decoded information frames by employing the network encoding technique at the BS. The output codewords of the CUs are denoted as: $\left\{\overleftrightarrow{\hat{X}_{r_{k}}^{n}}\right\}_{k=1}^{K}$, while those of the $k$ th CU during the $n$th frame may be expressed as:

$$
\hat{X}_{r_{k}}^{n}=M \cdot X_{s_{l}}^{n}=\left[\hat{I}_{l} \mid \hat{P}_{k}\right],
$$

where $X_{s_{l}}^{n}$ is the information frame transmitted from the $l$ th PU within the $n$th frame to both the CUs and to the BS. Additionally, the transfer matrix $M$ defined in [15] describes the corresponding network codes invoked for our DNC-CCR scheme. As seen in Fig. 3, the transfer matrix $M$ is comprised of two components, since we have $M=\left[I_{l} \mid P_{k}\right]$, where the identity matrix $\{I\}_{l}(l \in L)$, represents the sequences transmitted from the PUs during the broadcast phase, while the parity matrix $\{P\}_{l \times k}(k \in K)$ represents to the CUs' transmissions during the cooperative phase. Therefore, the corresponding entry $I_{l}$ in Fig. 3 represents the successful/unsuccessful reception of the information frame recovered at the BS during the broadcast phase, which obeys the following rule:

$$
I_{l}= \begin{cases}0, & \text { If } X_{s_{1}}^{n} \text { is not recovered successfully; } \\ 1, & \text { If } X_{s_{1}}^{n} \text { is recovered successful; }\end{cases}
$$

We note that the coefficient $p_{l, k}$ shown in Fig. 3 is gleaned from the transfer matrix of a linear block code defined over the Galois field $G F(|q|)$, where $|q|$ is the alphabet size ( $q=$ $2^{b}$ ), and $b$ is an integer higher than zero [16], [18]. The transfer matrix constructed over the $G F(|q|)$ is provided by the software application SAGE [36]. As shown in Fig. 3, the variable $p_{l, k}$ represents the specific transmission state, during which the information frame is transmitted from $\mathrm{PU}_{l}$ to $\mathrm{CU}_{k}$ as detailed below:

$$
p_{l, k}= \begin{cases}0, & \text { unsuccessful } \\ 1, & \text { successful }\end{cases}
$$

The network encoding process is represented by Eq (10), where we construct the parity frames $\hat{P}_{k}=\left[P_{1}, P_{2}, \ldots, P_{k-1}, P_{k}\right]$ from the $K$ CUs. Additionally, the parity frame transmitted by the $k$ th $\mathrm{CU}$ is given by:

$$
P_{k}=I_{1} p_{1, k} \oplus I_{2} p_{2, k} \oplus \cdots \oplus I_{l} p_{l, k} .
$$

As an illustration, we present a specific example associated with $L=2$ PUs and $K=2$ CUs, where we have orthogonal 


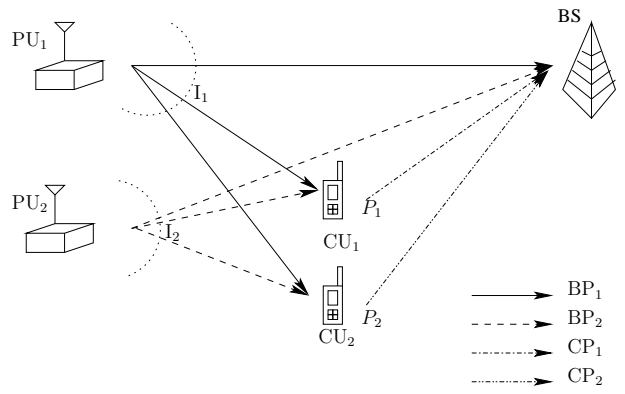

Fig. 4. The schematic of DNC-CCR scheme based $M_{2 \times 4}$ matrix. Two PUs intended to transmit their source messages $I_{1}$ and $I_{2}$, respectively.

channels among the pairs of PUs and CUs, as shown in Fig. 4. We assumed that each PU broadcasts $m_{1}=1$ information frame and then each $\mathrm{CU}$ transmits $m_{2}=1$ parity frame composed of the non-binary linear combinations of its information frames defined over $G F(|q|)$ to the BS. Then there are $\left(m_{1} \times L+m_{2} \times K\right)=4$ phases during a transmission session, including the pair of broadcast phases $B P_{1}$ and $B P_{2}$ as well as the pair of cooperative phases $C P_{1}$ and $C P_{2}$. The coding arrangement of this specific example can be summarized as follows:

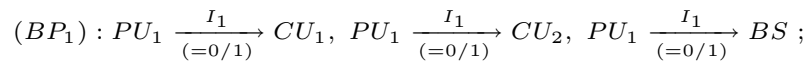

$$
\begin{aligned}
& \left(B P_{2}\right): P U_{2} \underset{(=0 / 1)}{\stackrel{I_{2}}{(=0 / 1}} C U_{1}, P U_{2} \underset{(=0 / 1)}{\stackrel{I_{2}}{\longrightarrow}} C U_{2}, P U_{2} \underset{(=0 / 1)}{\stackrel{I_{2}}{\longrightarrow}} B S ;
\end{aligned}
$$

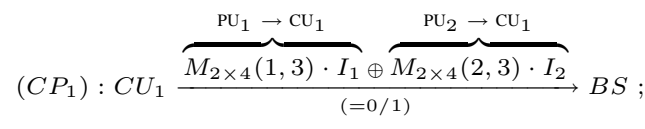

$$
\begin{aligned}
& \left(C P_{2}\right): C U_{2} \frac{\overbrace{M_{2 \times 4}(1,4) \cdot I_{1}}^{\mathrm{PU}_{1} \rightarrow \mathrm{CU}_{2}} \overbrace{(=0 / 1)}^{\mathrm{PU}_{2 \times 4}(2,4) \cdot I_{2}} \rightarrow \mathrm{CU}_{2}}{\longrightarrow} B S .
\end{aligned}
$$

Note that the arrow ' $\rightarrow$ ' represents the transmission direction. The notation $0 / 1$ below the right arrow of Eq. (17) indicates whether the transmission was successful or not, where $I_{l}$ is defined in Eq. (11), which $p_{l, k}$ in Eq. (12). The notation ' $\oplus$ ' represents the non-binary linear combination of the information frames. The corresponding transfer matrix $M_{2 \times 4}$ of [15] constructed for our specific system of Fig. 4 is defined as:

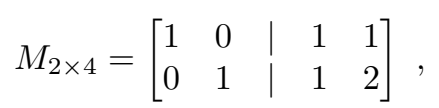

where the subscript of $M_{N_{r} \times N_{c}}$ represents the matrix having $N_{r}$ rows and $N_{c}$ columns. Observe by referring to Eq. (13) that the parity frame is transmitted from $\mathrm{CU}_{1}$ to the $\mathrm{BS}$ during $C P_{1}$ may be expressed as:

$$
P_{1}=M_{2 \times 4}(1,3) I_{1} \oplus M_{2 \times 4}(2,3) I_{2}=I_{1} \oplus I_{2} .
$$

Then the parity frame $P_{2}$ of from $\mathrm{CU}_{2}$ during $C P_{2}$ becomes:

$$
P_{2}=M_{2 \times 4}(1,4) I_{1} \oplus M_{2 \times 4}(2,4) I_{2}=I_{1} \oplus 2 I_{2},
$$

where $M_{2 \times 4}(i, j)$ represents row $i$ and column $j$ of the corresponding transfer matrix $M$, with $i \in[1,2]$ and $j \in$ $[1,2,3,4]$.

We also define the modified transfer matrix $M_{2 \times 4}^{\prime}$ with respect to the original transfer matrix $M_{2 \times 4}$, which takes into account the success/failure of each transmission during a specific transmission session. In order to detect the information frames of the PUs, the BS has to be aware of how each parity frame was constructed at the CUs. Hence the modified transfer matrix $M_{i \times j}^{\prime}$ is assumed to be known at the BS. If all the transmitted frames are successfully decoded, the modified transfer matrix is defined as $M_{2 \times 4}^{\prime}(i, j)=M_{2 \times 4}(i, j)$. As shown in Fig. 1, $\left(K \times m_{2}\right)$ parity frames are transmitted by the $K$ CUs, which contain the non-binary linear combinations of its own information frames along with the successfully decoded information frames received from the $L$ PUs. Let us denote the actual output codeword of the CUs as $\hat{X}_{r_{k}}^{\prime}{ }^{n}$ corresponding to the modified matrix $M_{i \times j}^{\prime}$. Hence $\hat{X}_{r_{k}}^{\prime}{ }^{n}$ contains all zeros, when the BS failed to successfully recover the $\left(L \times m_{1}\right)$ information frames or failed to receive the $\left(K \times m_{2}\right)$ parity frames, otherwise we have $\hat{X}_{r_{k}{ }^{n}}=\hat{X}_{r_{k}{ }^{n}}$.

\section{Principle of Adaptive Dynamic Network Coding}

In our ADNC scheme, we assume that the network-coding decoder at the BS is capable of sending back a feedback flag to the network encoders at the CU/RNs, as shown in Fig. 1. The transmission of the parity frames from the CUs is controlled by this feedback flag. Additionally, we conceive and analyze an efficient ADNC scheme using two methods, namely M1 and M2 associated with different amount of feedback requirements.

1) $A D N C-M 1$ : By referring to [16], the ADNC-M1 adaptively adjusts the number of frames transmitted from the CUs for each transmission session. The BS feeds back a single bit, $S_{f}$, following the reception of a set of $\left(L \times m_{1}\right)$ information frames from the $L$ PUs. If the CU/RNs received $S_{f}=0$, this implies that the BS has failed to correctly decode the information frames received from all the PU/SNs and hence the CU/RNs have to transmit $\left(K \times m_{2}\right)$ parity frames to the BS. Otherwise, if the BS successfully decoded the PU's information, the value of the feedback flag is set to $S_{f}=1$. Let us denote the number of CUs invoked in an actual transmission by employing the ADNC technique during the cooperative phase as $K^{\prime}$. The actual number of information frames, $K^{\prime} m_{2}$, transmitted from the $K$ CUs by the ADNC-M1 technique obeys the following rules:

$$
K^{\prime} m_{2}= \begin{cases}0, & S_{f}=1 ; \\ K m_{2}, & S_{f}=0 ;\end{cases}
$$

where $m_{2}$ denotes the number of information frame transmitted per $\mathrm{CU}$.

2) $A D N C-M 2:$ In contrast to ADNC-M1, in the ADNC-M2 scheme we assume that the BS feeds back $L$ bits associated with the $L$ PUs, namely $S_{f_{l}}$, to the CUs. Additionally, if the CUs received $\sum_{l=1}^{L}\left(S_{f_{l}}\right)=0$, this implies that the BS has failed to correctly decode all the information packets received from the PUs and hence the CUs have to transmit the same number of parity frames to the BS. Otherwise, the CUs do not have to transmit, provided that we have $\sum_{l=1}^{L}\left(S_{f_{l}}\right)=1$, which indicates that the BS has indeed succeeded in flawlessly decoding all PUs' frames. Moreover, if most of the PUs' information frames are successfully received by the BS, except for the failed detection of $\vartheta$ PUs, then the CU may only have to transmit $\vartheta m_{2}$ number of parity frames to the BS. Hence the number of parity frames required can be calculated by counting the specific number of the feedback flags indicating 
successful reception by the BS. More specifically, if the BS successfully received some of the information frames from the PUs, it will send a feedback flag to CUs, which will hence retransmit the failed information frames to the BS. Hence the adaptive configuration of the actual number of $K^{\prime}$ CUs' information frames obeys the following rules:

$$
K^{\prime} m_{2}= \begin{cases}0, & \sum_{l}^{L}\left(S_{f_{l}}\right)=1 ; \\ K m_{2}, & \sum_{l}^{L}\left(S_{f_{1}}\right)=0 ; \\ \vartheta m_{2}, & \text { Otherwise; }\end{cases}
$$

where $\vartheta$ denotes the number of CUs that have to relay the PU's information to the BS, $\vartheta \in L$. For example, the value of $\vartheta$ in our $M_{2 \times 4}$-based scheme is 1 , which implies that the BS failed to successfully decode a single PU's transmission. Additionally, in an $M_{N_{r} \times N_{c}}$-based scheme, the value of $\vartheta$ is given by $(K-i)$. The CUs would relay the required $\vartheta$ parity frames to the BS during the cooperative phase. The system's achievable performance will be discussed in Section IV.

\section{SyStem DESIGN}

In this section, the design guidelines of our proposed system will be discussed first. Then the transmission rates of both ADNC-M1 and ADNC-M2 are analysed. Based on our investigations of both the network coding rate and the diversity order of our ADNC-M1 and ADNC-M2 schemes, we decide upon our final recommended prototype system.

\section{A. Adaptive TTCM transmission scenario}

All transmission links shown in Fig. 1 have employed the ATTCM scheme detailed in Eq. (8) and Eq. (9), where each link may employ different modulation modes during the entire transmission period. Under the DNC policy of Section II-B, the PUs (or SNs) transmit at the same rate. We consider the "worst" PU, whose link towards both the CUs and the BS has the lowest SNR, which forces some of the PUs which actually experience a high SNR to transmit at a rate lower than their own affordable rate. This allows the CU to perform bit-by-bit combination of the decoded information. Thus, the lowest-rate, but most resilient modulation modes are activated for all the $L \mathrm{SD}$ and $L \mathrm{SR}$ links, which also affects the $(L \times K) \mathrm{SR}$ links. The rate achievable at the SNs of our proposed scheme during the broadcast phases can be written as:

$$
R_{T x}^{P U_{l}}=\min \left\{C_{s_{l} d}^{*}, C_{s_{l} r_{k}}^{*}\right\}, \quad l \in L, k \in K .
$$

As for the $K \mathrm{RD}$ links, each link obeys the adaptive modulation mode selection rule of Eq. (8) and Eq. (9). Additionally, the achievable rate of the $\mathrm{CU} / \mathrm{RN}$ to $\mathrm{BS}$ link during the cooperative phases is given by:

$$
R_{T x}^{C U_{k}}=\max \left\{C_{r_{k} d}^{*}\right\}, \quad k \in K,
$$

where the values of $C_{s_{l} d}^{*}, C_{s_{l} r_{k}}^{*}$ and $C_{r_{k} d}^{*}$ can be obtained from Eq. (8), depending on the instantaneous channel conditions. Our proposed scenario achieves a FER lower than the $10^{-3}$ target at the $\mathrm{RN}$, in order to minimize the potential error propagation from the CUs to the BS. The strategy defined in Eq. (21) during the broadcast phase is not the only scenario we have investigated. We have also tested another promising scenario, which select the best PU that has the highest SNR among all PUs, when the achievable rate is defined as: $R_{T x}^{P U_{l}}=\max \left\{C_{s_{l} d}^{*}, C_{s_{l} r_{k}}^{*}\right\}$. However, the corresponding FER performance becomes worse than our target of $10^{-3}$, which leads to an excessive probability of errors at the CUs. Hence, we ultimately recommend the better strategy of Eq. (21).

\section{B. Analysis of Transmission Rate}

In this section, we investigate the attainable transmission rate of our proposed schemes. We assume that we have $N$ frame sessions and the length of each TS in the frame is T. As discussed in Section II, this TS is split into two parts, yielding $T=T_{1}+T_{2}$, where $T_{1}$ is used for the PU's transmission during the broadcast phase and $T_{2}$ is allocated for the CUs to relay the combined information of both the PU and $\mathrm{CU}$ during the cooperative phase. If the PU activates a higherthroughput modulation mode, then the SNR required by the PU will be high. As a benefit, this will shorten the PU's transmission duration of $T_{1}$. Consequently, this would grant a longer transmission period for the CUs. Thus, we increase $T_{2}$, when $T_{1}$ is reduced. Moreover, we assumed that the PU and CU have the same Baud rate (symbol rate) of $R_{s}^{P U}=R_{s}^{C U}$ symbol/s during the entire transmission period. Meanwhile, the number of bits per frame of the PU and CU are the same, namely $N_{b}^{P U}=N_{b}^{C U}$ bits. Then the number of bits during the broadcast phase is given by:

$$
N_{b}^{P U}=\frac{L R_{s}^{P U}}{R_{T x}^{P U_{l}}},
$$

while during the cooperative phase, it is:

$$
N_{b}^{C U}=\frac{K^{\prime} R_{s}^{C U}}{R_{T x}^{C U_{k}}},
$$

where $K^{\prime}$ was defined in Section II-C.2. Then the Baud rate of the PU is $R_{s}^{P U}=N_{b}^{P U} / T_{1}$, while that of the CU is $R_{s}^{C U}=$ $N_{b}^{C U} / T_{2}$.

In our proposed DNC-CCR scheme of Section II-B, the TSs are shared by the PU and CU. We assumed that the amount of information to be transmitted by the PU and CU is identical, thus we have:

$$
\frac{L R_{s}^{P U}}{R_{T x}^{P U_{l}} T_{1}}=\frac{K^{\prime} R_{s}^{C U}}{R_{T x}^{C U_{k}} T_{2}} .
$$

Then, based on Eq. (25), the relationship between $T_{1}$ and $T_{2}$ is given by:

$$
\frac{T_{2}}{T_{1}}=\frac{K^{\prime} R_{T x}^{P U_{l}}}{L R_{T x}^{C U_{k}}} .
$$

Since $T_{2}=T-T_{1}$, based on Eq. (26), we have

$$
T_{1}=\frac{L R_{T x}^{C U_{k}}}{K^{\prime} R_{T x}^{P U_{l}}+L R_{T x}^{C U_{k}}} T .
$$

The average throughput of the entire system may be quantified by the ratio of the total number of transmitted information bits divided by the number of transmission TSs. This metric is similar to the concept of the overall rate defined in [27, (18)]. We also assume that our proposed system transmits its 
messages at the same rate during each $N$-frame session. Then the overall throughput per frame per user for our proposed DNC-CCR scheme is given by:

$$
\begin{aligned}
\eta_{D N C} & =\frac{1}{N} \sum_{n=1}^{N} \frac{\sum_{l=1}^{L} R_{T x}^{P U_{l}} T_{1}}{L \times T}, \\
& =\frac{1}{N} \sum_{n=1}^{N} \frac{\sum_{l=1}^{L} \frac{L R_{T x}^{P U_{l}} R_{T x}^{C U_{k}}}{\left(K^{\prime} R_{T x}^{P U_{l}}+L R_{T x}^{C U_{k}}\right)}}{L \times T},
\end{aligned}
$$

where again, $N$ denotes the number of frames and $L$ is the total number of PUs. In our $M_{2 \times 4}$-based system, the number of frames transmitted from the PU is $L=2$, while, the number of information frames transmitted is $L=4$ for the $M_{4 \times 8^{-}}$ based system.

1) ADNC scheme: Let us now consider the achievable transmission rate of our proposed ADNC-CCR scheme. If all the PUs transmit all their messages to the BS successfully, the CUs do not have to relay the source information to the BSs. By contrast, the CUs will relay the source information to the BS, if the transmissions from the PU to the BS failed. Thus we can obtain the time allocations between PUs and CUs as follows:

$T_{1}\left\{\begin{array}{l}=T, T_{2}=0 ; \text { If the PU's transmission is successful. } \\ =\frac{L R_{T x}^{C U_{k}}}{K^{\prime} R_{T x}^{P U_{l}}+L R_{T x}^{C U_{k}}} T, T_{2}=T-T_{1} ; \text { Otherwise; }\end{array}\right.$

where $K^{\prime}$ is different in the ADNC-M1 scheme of Section IIC. 1 and in the ADNC-M2 scheme of Section II-C.2. Moreover, if the PU transmitted successfully to the BS, we can save the entire $T_{2}$ duration for the CU's communication. However, if the PU failed to successfully transmit its message to the BS, then the CU would relay the PU's message to the BS. If the transmit SNR of the PU is higher, the relaying period of the $\mathrm{CU}$ will be reduced and this allows the system to grant a longer transmit-duration for the CUs. By contrast, at a low SNR the opposite trend prevails. When the PU's transmission is successful, the average throughput of $L$ PUs relying on our ADNC-CCR scheme is given by:

$$
\begin{aligned}
\eta_{A D N C} & =\frac{1}{N} \sum_{n=1}^{N} \frac{\sum_{l=1}^{L} R_{T x}^{P U_{l}} T}{L T} \\
& =\frac{1}{N} \sum_{n=1}^{N} \frac{\sum_{l=1}^{L} R_{T x}^{P U_{l}}}{L}
\end{aligned}
$$

If the PU's transmission failed, then the CU/RN will transmit the PU's information to the BS. The overall throughput takes into consideration both the PU's information and the CU's own information, where we have $\eta_{A D N C}=\eta_{D N C}$, when the PU's transmission failed, where $\eta_{D N C}$ is detailed in Eq. (29). Hence, the overall throughput per frame per user of the proposed ADNC scheme is given by:

$\eta_{A D N C}= \begin{cases}\frac{1}{N} \sum_{n=1}^{N} \frac{\sum_{l=1}^{L} R_{T x}^{P U_{l}}}{L} & \text { PU's transmission was successful; } \\ \frac{1}{N} \sum_{n=1}^{N} \frac{\sum_{l=1}^{L} R_{T x}^{P U_{l}} T_{1}}{L \times T} & \text { PU's transmission failed; }\end{cases}$

where $T_{1}$ is defined in Eq. (27).
2) Direct Transmission: The overall transmission rate per frame per user of the non-cooperative scheme recorded for the whole TS duration $T$ becomes:

$$
\eta_{S D}=\frac{1}{N} \sum_{n=1}^{N} \frac{\sum_{l=1}^{L} R_{T x}^{P U_{l}}}{L}
$$

Finally, we will compare the overall performance of these two systems in Section IV.

\section{Diversity Order and Network Code Rate}

The network coding rate $R_{N C}$ characterising the multiplexing capability of the network coding scheme exemplified in Section II-B may be expressed as:

$R_{N C}=\frac{\text { Total number transmitted information frames of PUs }}{\text { Total number of information frames of PUs and CUs }}$

By further considering the ADNC scheme detailed in Section II-C, the network code rate of ADNC-M1 is given by:

$$
R_{N C}^{A D N C-M 1}= \begin{cases}\frac{L m_{1}}{L m_{1}}, & S_{f}=1 \\ \frac{L m_{1}}{L m_{1}+K m_{2}}, & S_{f}=0\end{cases}
$$

Moreover, the network coding rate of the ADNC-M2 scheme may be expressed as:

$$
R_{N C}^{A D N C-M 2}= \begin{cases}\frac{L m_{1}}{L m_{1}}, & \sum_{l}^{L}\left(S_{f_{l}}\right)=1 ; \\ \frac{L m_{1}}{L m_{1}+K m_{2}}, & \sum_{l}^{L}\left(S_{f_{l}}\right)=0 ; \\ \frac{L m_{1}}{L m_{1}+\vartheta m_{2}}, & \text { Otherwise; }\end{cases}
$$

where the number of CUs is assumed to be the same as the number of PUs in our design. As shown in Eq. (33) and Eq. (34), the resultant network coding rate is: $R_{N C}^{A D N C}=$ $\frac{L m_{1}}{L m_{1}}=1$, while the value of $K m_{2}$ can be adaptively adjusted toward 0 in order to increase the achievable multiplexing gain. In order to highlight our generic design principles, the $M_{4 \times 8^{-}}$ based system is taken into consideration. The transfer matrix $M_{4 \times 8}$ is provided by [16], [17]:

$$
M_{4 \times 8}=\left[\begin{array}{cccc|cccc}
1 & 0 & 0 & 0 & 3 & 7 & 3 & 6 \\
0 & 1 & 0 & 0 & 5 & 7 & 7 & 4 \\
0 & 0 & 1 & 0 & 2 & 4 & 6 & 1 \\
0 & 0 & 0 & 1 & 5 & 5 & 3 & 2
\end{array}\right] .
$$

Then we have four broadcast phases and four cooperative phases. The construction of the parity frames $P_{k}$ of the $k$ th $\mathrm{CU}$ is detailed in Eq. (13). Moreover, the modified transfer matrix $M^{\prime}$ of the actual communication is given by $M_{4 \times 8}^{\prime}(i, j)=M_{4 \times 8}(i, j)$, provided that all transmitted frames are successfully decoded by the BS. Referring to Fig. 1, the network encoders of the CUs generate the parity frames based on the information frames. The network decoders at the BS will decode the parity frames based on the modified matrix introduced in Section II-B. Therefore, the $M_{2 \times 4}$-based scheme and the $M_{4 \times 8}$-based scheme are comparable, since they share the same parameter values of $R_{N C}^{A D N C}=\frac{L m_{1}}{L m_{1}+K m_{2}}=\frac{1}{2}$, while $S_{f}=0$ or $\sum_{l}^{L}\left(S_{f_{l}}\right)=0$ refer to Eq. (33) and Eq. (34), respectively.

Accordingly, the information rate $R_{\text {info }}$ of our proposed system can be expressed as [17]:

$$
R_{\text {info }}=R_{N C} \times \eta_{A D N C},
$$


which is near-instantaneously time-variant [37], [38], since it may be changed for each transmission session. Furthermore, the diversity order reflecting the degree of space diversity gain attained by employing our network coding aided system may be expressed as [39]:

$$
D_{A D N C}=K^{\prime}+m_{2}
$$

where the value of $K^{\prime}$ is $K^{\prime} \in[0,4]$ for the ADNC-M1 scheme and $K^{\prime} \in[0,1,2,3,4]$ for the ADNC-M2 scheme, when $M_{4 \times 8}$ is used. Additionally, the maximum and minimum value of $D_{A D N C-M 1}$ is identical to that of $D_{A D N C-M 2}$, but the diversity order $D_{A D N C-M 1}$ is higher than in the scenario $D_{A D N C-M 2}$, when the BS fails to recover the PU's information, since the value of $K^{\prime}$ in the $D_{A D N C-M 2}$ scheme can be $[1,2,3]$. Fig. 5 shows both the FER performance and the average per-user per-link throughput versus $E_{b} / N_{0}$ for our ATTCM aided ADNC assisted CCR system based on the $M_{4 \times 8}$ matrix. It can be seen in Fig. 5 that the FER performance of ADNC-M1 is better than that of the ADNC-M2, since it has a higher diversity order in some situations, when their throughputs are comparable. In our CCR

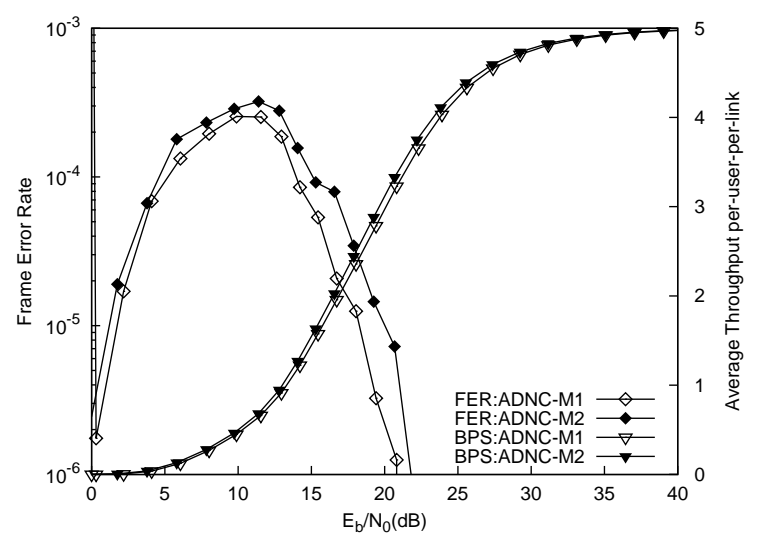

Fig. 5. The FER performance and average throughput per user per link versus $E b / N_{0}$ of the proposed ATTCM aided ADNC scheme based on the $M_{4 x 8}$ matrix detailed in Section III, as well as of ADNC-M1 for $K^{\prime} \in 0,4$, and ADNC-M2 for $K^{\prime} \in 0,1,2,3,4$.

scheme, we aim for reducing the bandwidth requirement, while increasing the transmission rate of the PUs. We will employ ANDC-M2 in the investigations of Section IV because it has a higher multiplexing gain.

\section{NUMERICAL RESUltS AND DISCUSSIONS}

Fig. 2 shows the FER performance of the five individual non-adaptive TTCM modes of Eq.(8), when communicating over uncorrelated Rayleigh fading channels as well as the FER of TTCM-aided DNC and of the corresponding noncooperative schemes, when transmitting over our combined ${ }^{1}$ quasi-static (shadow) and Rayleigh (fast) fading channels. Fig. 2 substantiates that the curves that consider only an uncorrelated Rayleigh fading channel always exhibit a better performance than the curves that characterise the combined quasi-static and Rayleigh fading scenarios. It is observed that

\footnotetext{
${ }^{1}$ When the channel gain incorporates both the shadow- and fast-fading components, the adaptive transmission regime counteracts the shadow fading, but it is typically unable to accommodate the Rayleigh-fading.
}

the FER performance of our ATTCM aided DNC scheme is always better than that of the non-cooperative scheme, regardless of the specific modulation modes. Naturally, the 4PSK modulation mode has the best FER performance. It is shown in Fig. 2 that the DNC-4PSK arrangement attains an approximately $20 \mathrm{~dB}$ gain in comparison to the SD-4PSK scheme at $F E R=10^{-3}$. As expected, the 64QAM mode has the worst FER performance, when viewing this comparison for an SNR-perspective, the DNC-64QAM scheme required an extra $12 \mathrm{~dB}$ power compared to both DNC-4PSK and to DNC-64QAM.

We define $E_{b} / N_{0}$ as the transmitted energy per bit to noise power spectral density ratio:

$$
E_{b} / N_{0} \mathrm{~dB}=\gamma_{t} \mathrm{~dB}-10 \log _{10}\left(R_{\text {info }}\right),
$$

where $\gamma_{t}$ is the transmit $S N R^{2}$ and $R_{\text {info }}$ is the system's achievable throughput, which is defined in Eq. (36).

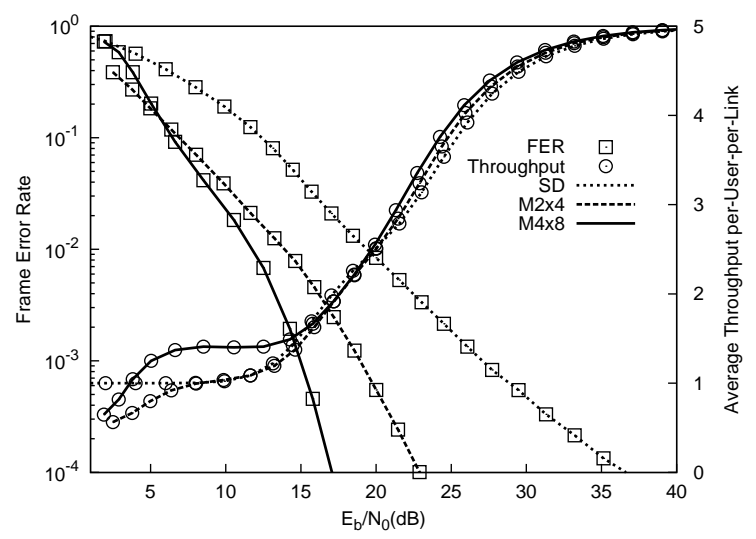

Fig. 6. The FER performance and average throughput per user per link versus $E b / N 0_{t}$ of the proposed System 2 of ATTCM aided ADNC scheme refer to Table I. "M2x4" and "M4x8" represents the FER and average throughput of our ATTCM aided ADNC scheme activating one of five channel quality dependent modulation modes using $10^{6}$ frames based on the matrix $M_{2 \times 4}$ and $M_{4 \times 8}$, respectively.

Fig. 6 shows the FER versus $E_{b} / N_{0}$ performance of the $M_{2 \times 4}$ and $M_{4 \times 8}$-based System 2 employing the ADNC arrangement, which represents the practical ATTCM transmission scheme of Table I, as introduced in Section II-A. Its performance is benchmarked against that of the ATTCM aided non-cooperative communication scheme. As observed in Fig. 6, the $M_{4 \times 8}$-based scheme is capable of providing a significant $E_{b} / N_{0}$ performance improvement of $19 \mathrm{~dB}-15 \mathrm{~dB}=$ $4 \mathrm{~dB}$ at $F E R=10^{-3}$ in comparison to the $M_{2 \times 4}$-based scheme. In comparison to the non-cooperative schemes, our proposed $M_{4 \times 8}$ and $M_{2 \times 4}$-based ADNC arrangement has a better FER performance. More specifically, our $M_{4 \times 8}$-based scheme attains an approximately $27 \mathrm{~dB}-15 \mathrm{~dB}=8 \mathrm{~dB}$ gain compared to its non-cooperative counterpart at $\mathrm{FER}=10^{-3}$.

As we discussed in Section III-B, the average throughput

\footnotetext{
${ }^{2}$ The concept of transmit SNR [32] is unconventional, as it relates quantities to each other at two physically different locations, namely the transmit power to the noise power at the receiver, which are at physically different locations. However, this is a computationally convenient definition.
} 


\begin{tabular}{|c|r|r|}
\hline System & System 1 & System 2 \\
\hline The output $C^{*}\left(\gamma_{R}<\gamma_{0}\right)$ & case 1 (No transmission) & case 2 (TTCM-4PSK) \\
\hline Principle of ADNC & ADNC-M2 & ADNC-M2 \\
\hline Channel & Rayleigh and Quasi static fading channel & Rayleigh and Quasi static fading channel \\
\hline Number of frames $N$ & $10^{6}$ & $10^{6}$ \\
\hline Adaptive Coding & ATTCM & ATTCM \\
\hline Modulation & Q-PSK, 8-PSK, 16-QAM, 32-QAM, 64-QAM & Q-PSK, 8-PSK, 16-QAM, 32-QAM, 64-QAM \\
\hline FER bound & $10^{-3}$ & $10^{-3}$ \\
\hline Transfer matrix of DNC & $M_{2 \times 4}, M_{4 \times 8}$ & $M_{2 \times 4}, M_{4 \times 8}$ \\
\hline Number of PUs & $L=2,4$ & $L=2,4$ \\
\hline Number of CUs & $K=2,4$ & $K=2,4$ \\
\hline$m_{1}$ [frame] & 1 & 1 \\
\hline$m_{2}$ [frame] & 1 & 1 \\
\hline Pathloss exponent $\alpha$ & 3 & 3 \\
\hline
\end{tabular}

TABLE I

THE MAIN PARAMETERS OF OUR TWO SYSTEMS, WHERE SYSTEM 1 INCLUDES A NO TRANSMISSION MODE, WHILE SYSTEM 2 WOULD INVOKE THE 'TTCM-4PSK' MODE WHEN THE CHANNEL QUALITY IS POOR.

per user of the entire system can be summarized as:

$$
\bar{\eta}_{\text {ave }}=\underbrace{\frac{\overbrace{L R_{\text {tran }}^{\text {PU }} T_{\text {broadcast }}}^{\text {Transmitted packet of PU }}}{\underbrace{}_{\text {broadcast }}+K T_{\text {cooperative }}}}_{\text {Required orthogonal channel }}=\frac{L R_{\text {tran }}^{\text {PU }}}{L+K} .
$$

Explicitly, Fig. 6 illustrates the average throughput per-user per-link versus $E_{b} / N_{0}$ performance of the $M_{2 \times 4}$ and $M_{4 \times 8^{-}}$ based ATTCM-ADNC-CCR scheme, when we employed the five TTCM modes of Eq.(8) and used the case 2 scenario of Eq. (9). In contrast to the ADNC arrangement, at low SNRs the non-cooperative arrangement has a throughput of 1 iBPS. It is observed in Fig. 6 that the throughput trends of these three schemes are similar and they approach $5 \mathrm{iBPS}$ at $E_{b} / N_{0}=$ $40 \mathrm{~dB}$. Therefore, the $M_{4 \times 8}$-based scheme has the best FER performance at a comparable throughput.

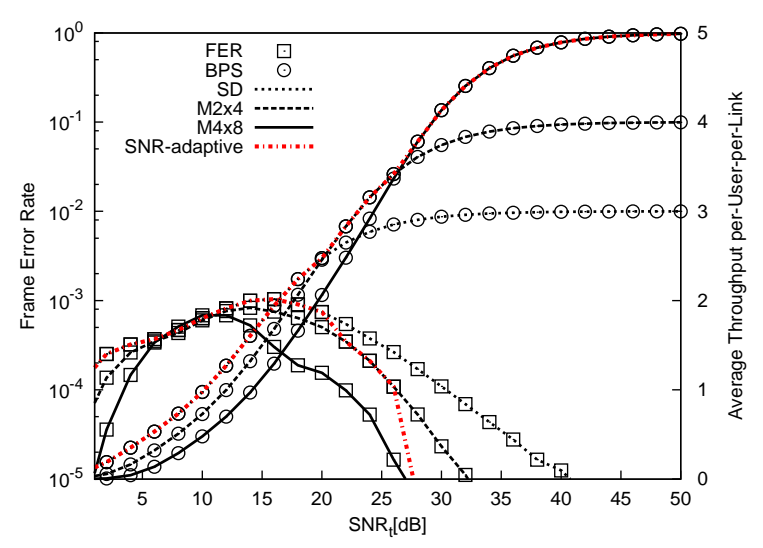

Fig. 7. The FER performance and average throughput per user per link versus SNR $t$ of the proposed System 1 of ATTCM aided ADNC scheme refer to Table I. "SD", "M2x4" and "M4x8" represents the FER and average throughput of our ATTCM aided non-cooperative scheme using one of three modulation modes and the ADNC scheme for four and five modulation modes based on matrix $M_{2 \times 4}$ and $M_{4 \times 8}$, respectively. "SNR-adaptive" ATTCMADNC-CCR scheme is represented that always activates the best scheme for the set of "SD", "M2x4" and "M4x8" based schemes in terms of the average throughput as a function of the SNR which has described in Section IV.The number of transmitted frames is $10^{6}$.

Fig. 7 shows the FER versus $S N R_{t}$ performance of $M_{2 \times 4}$ and $M_{4 \times 8}$-based System 1 employing the ADNC arrangement, which the parameters are shown in Table I. In Fig. 7, we compare the throughput of three schemes at a comparable FER. Explicitly, the non-cooperative scheme employed the

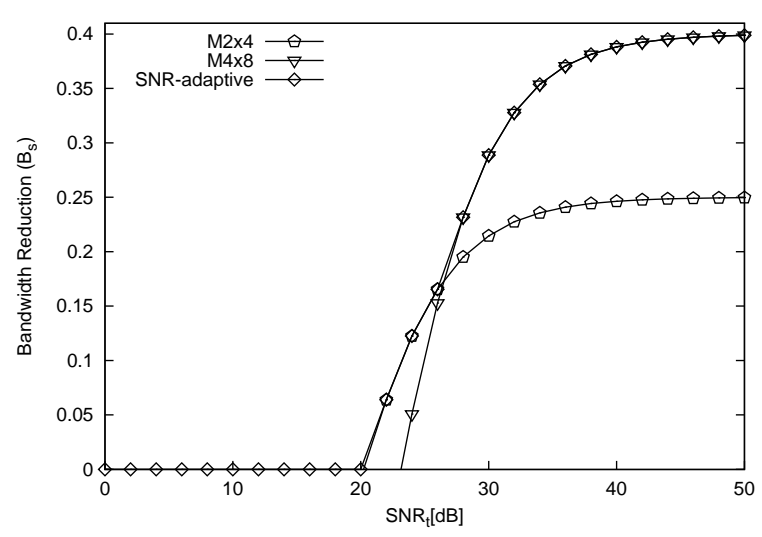

Fig. 8. The bandwidth reduction $B_{s}$ versus $\mathrm{SNR}_{t}$ of the proposed System 1 of ATTCM aided ADNC scheme refer to Table. I. "SD", "M2X4" and "M4x8" are denoted the FER and average throughput of ATTCM aided non-cooperative scheme using one of three modulation modes and the ADNC scheme for four and five modulation modes based on matrix $M_{2 \times 4}$ and $M_{4 \times 8}$, respectively. "SNR-adaptive" ATTCM-ADNC-CCR scheme is represented that always activates the best scheme for the set of "SD", "M2 4 " and "M4x8" based schemes in terms of the average throughput as a function of the SNR which has described in Section IV.

BPSK, 8PSK, 16QAM of ATTCM transmission modes. The reason for considering three modulation modes for our noncooperative scheme is because the FER performance of the non-cooperative benchmark scheme is somewhat poor. If a 32QAM modulation mode were to be used, the FER would become excessive. Hence we opted for four modulation modes for our ATTCM-ADNC-CCR $M_{2 \times 4}$ matrix. Observe in Fig. 7 that the FER recorded at the RN becomes lower than $10^{-3}$ both for the ADNC and for the non-cooperative scheme. The FER performance curves of the $M_{2 \times 4}$ and of the $M_{4 \times 8}$-based ATTCM-ADNC-CCR scheme and of the non-cooperative scheme crossed each other at $S N R_{t}=10 \mathrm{~dB}$. Beyond that point, the FER performance of the ADNC scheme became better than that of the non-cooperative scheme, namely for $\mathrm{SNR}_{t}>11 \mathrm{~dB}$. Moreover, at $\mathrm{FER}=10^{-5}$ our proposed $M_{4 \times 8^{-}}$ based scheme attains an $40 \mathrm{~dB}-26.5 \mathrm{~dB}=13.5 \mathrm{~dB}$ gain compared to the non-cooperative scheme.

As observed in Fig. 7, our proposed $M_{4 \times 8}$-based ADNC scheme has a better throughput than the non-cooperative scheme for $S N R_{t}>25 \mathrm{~dB}$. Explicitly, we found in Fig. 7 that the ADNC scheme achieved a throughput of 5.0 iBPS, which is $5.0-3.0=2.0$ bits higher than that of the non-cooperative 
scheme at $\mathrm{SNR}_{t}=45 \mathrm{~dB}$. Observe furthermore in Fig. 7 that the throughput of the $M_{2 \times 4}$-based ADNC scheme is higher than that of the non-cooperative scheme for $S N R_{t}>20 \mathrm{~dB}$. More specifically, the proposed $M_{4 \times 8}$-based ADNC scheme requires four TSs, while the $M_{2 \times 4}$-based scheme only requires two TSs, when the BS failed to flawlessly receive the source information from the $\mathrm{SN}$, albeit these ADNC schemes failed to achieve a higher throughput for low $\mathrm{SNR}_{t}$. Hence, it is better to activate a non-cooperative model at a low SNR, since its throughput per user per link is better. Then the "SNR-adaptive" ATTCM-ADNC-CCR system characterized in Fig. 7 is the scheme that always activates the best scheme for the set of non-cooperative $M_{2 \times 4^{-}}$and $M_{4 \times 8^{-}}$based systems in terms of the average throughput as a function of the SNR. Note that at FER $=10^{-4}$ the FER performance of the "SNR-adaptive" scheme is better than that of the $M_{2 \times 4}$-based arrangement, but at FER $=10^{-5}$ worse than that of the $M_{4 \times 8}$ based scheme.

The bandwidth-reduction factor $\left(B_{s}\right)$ may be formulated as [20]:

$$
B_{s}=1-\frac{\eta_{S D}}{\bar{\eta}_{\text {ave }}},
$$

where $\eta_{S D}$ denotes the throughput of the non-cooperative system employing ATTCM as defined in Eq. (31) and $\bar{\eta}_{\text {ave }}$ is defined in Eq. (39).

In Fig. 8, we consider the attainable bandwidth reduction versus $\mathrm{SNR}_{t}$ for our ATTCM-ADNC-CCR system based on the $M_{2 \times 4}$ and $M_{4 \times 8}$ matrices of Table I. It is observed in Fig. 8 that the highest bandwidth reduction is achieved by the $M_{4 \times 8}$-based ADNC scheme, which result in $B_{s}=40 \%$ for $S N R_{t} \geq 42 \mathrm{~dB}$. Observe in Fig. 7 that the corresponding maximum throughput of the ADNC scheme is $\eta_{A D N C}=5$, while that of the non-cooperative scheme is $\eta_{S D}=3$. Thus, based on Eq. (40), we arrive at $B_{s}=1-\frac{3}{5}=0.4=40 \%$. By referring to Fig. 8 , we observe that the $M_{2 \times 4}$-based scheme may achieve $B_{s}=25 \%$ at high SNRs, namely for $S N R_{t} \geq 42 \mathrm{~dB}$. Thus, the $M_{4 \times 8}$-based ADNC scheme is capable of saving $40 \%-25 \%=15 \%$ more bandwidth than the $M_{2 \times 4}$-based ADNC scheme. Moreover, the performance of the "SNR-adaptive" ATTCM-ADNC-CCR scheme characterized in Fig. 8 provides the highest bandwidth for the PU, regardless of the SNR. In our proposed ADNC-CCR schemes, the size of the DNC matrix and the number of frames transmitted per PU/CU would influence the number of supported PUs. More specifically, based on a $\left(N_{r} \times N_{c}\right)$-element DNC matrix $M_{N_{r} \times N_{c}}$, the number of PUs that can be supported over an $N_{r}$-frame periods is given by $N_{r} / N_{f}$, where $N_{f}$ is the number of frames transmitted per PU during the $N_{r}$-frame period. Hence, the $M_{4 \times 8}$-based DNC-CCR scheme is capable of supporting $L=\{1,2,4\}$ PUs, when we have $N_{f}=\{4,2,1\}$, respectively. We found that both the FER and the average throughput per user per link versus $S N R_{t}$ performance would be similar, when supporting various number of PUs when using the same transfer matrix. Investigations based on a larger DNC matrix, such as the $M_{6 \times 12}$ of [40], will be considered in our future work.

\section{CONCLUSiOnS}

An ATTCM aided ADNC assisted CCR scheme was conceived, where both the ATTCM modulation mode and the
ADNC technique were configured according to the nearinstantaneous channel conditions. We found that the proposed ADNC aided CCR scheme enables the PU to either transmit 2 iBPS more information at a given SNR, or releasing up to $40 \%$ of bandwidth for exploitation by the CUs.

\section{REFERENCES}

[1] F. K. Jondral, "Cognitive radio: A communications engineering view," IEEE, Wireless Communications, vol. 14, pp. 28 -33, August 2007.

[2] J. Mitola and G. Q. Maguire, "Cognitive radio: making software radios more personal," IEEE Personal Communications, vol. 6, pp. $13-18$, Aug 1999.

[3] N. Devroye, P. Mitran, and V. Tarokh, "Achievable rates in cognitive radio channels," IEEE Transactions on, Information Theory, vol. 52, pp. 1813 - 1827, May 2006.

[4] B. Wang and K. Liu, "Advances in cognitive radio networks: A survey," IEEE Journal of Selected Topics in Signal Processing,, vol. 5, no. 1, pp. 5-23, 2011.

[5] A. Goldsmith, S. Jafar, I. Maric, and S. Srinivasa, "Breaking spectrum gridlock with cognitive radios: An information theoretic perspective," Proceedings of the IEEE, vol. 97, pp. 894 -914, May 2009.

[6] K. B. Letaief and W. Zhang, "Cooperative communications for cognitive radio networks," Proceedings of the IEEE, vol. 97, pp. 878 -893, May 2009.

[7] Y. Li, "Distributed coding for cooperative wireless networks: An overview and recent advances," IEEE Communications Magazine, vol. 47, pp. $71-77$, August 2009.

[8] K. Loa, C.-C. Wu, S.-T. Sheu, Y. Yuan, M. Chion, D. Huo, and L. Xu, "IMT-advanced relay standards [WiMAX/LTE Update]," IEEE Communications Magazine, vol. 48, pp. 40 -48, Aug 2010.

[9] D. Hwang, J. Choi, B. Clerckx, and G. Kim, "Mimo precoder selections in decode-forward relay networks with finite feedback," IEEE Transactions on Communications, vol. 59, pp. 1785 -1790, July 2011.

[10] Z. Ding, K. Leung, D. Goeckel, and D. Towsley, "Opportunistic relaying for secrecy communications: Cooperative jamming vs. relay chatting," IEEE Transactions on Wireless Communications, vol. 10, pp. 1725 1729, June 2011.

[11] T. Korakis, M. Knox, E. Erkip, and S. Panwar, "Cooperative network implementation using open-source platforms," IEEE Communications Magazine, vol. 47, pp. 134 -141, Feb. 2009.

[12] L. Chen, R. Carrasco, and I. Wassell, "Opportunistic nonorthogonal amplify-and-forward cooperative communications," Electronics Letters, vol. 47 , pp. $626-628$, Dec. 2011.

[13] M. Yassaee and M. Aref, "Slepian Wolf Coding Over Cooperative Relay Networks," IEEE Transactions on Information Theory, vol. 57, pp. 3462 -3482 , June 2011.

[14] F. Tian, W. Zhang, W.-K. Ma, P. Ching, and H. Poor, "An effective distributed space-time code for two-path successive relay network," IEEE Transactions on Communications, vol. 59, pp. 2254 -2263, Aug. 2011.

[15] M. Xiao and M. Skoglund, "Multiple-user cooperative communications based on linear network coding," IEEE Transactions on Communications, vol. 58, no. 12, pp. 3345-3351, 2010.

[16] J. Rebelatto, B. Uchoa-Filho, Y. Li, and B. Vucetic, "Adaptive distributed network-channel coding," IEEE Transactions on Wireless Communications, vol. 10, no. 9, pp. 2818-2822, 2011.

[17] J. Rebelatto, B. Uchoa-Filho, Y. Li, and B. Vucetic, "Multiuser cooperative diversity through network coding based on classical coding theory," IEEE Transactions on Signal Processing, vol. 60, no. 2, pp. 916-926, 2012.

[18] M. Xiao, J. Kliewer, and M. Skoglund, "Design of network codes for multiple-user multiple-relay wireless networks," IEEE Transactions on Communications, vol. 60, no. 12, pp. 3755-3766, 2012.

[19] W. Liang, S. X. Ng, and L. Hanzo, "Adaptive turbo trellis coded modulation aided cooperative cognitive radio," in IEEE Wireless Communications and Networking Conference (WCNC), pp. $2362-2366$, April 2012.

[20] W. Liang, S. X. Ng, and L. Hanzo, "Cooperative communication between cognitive and primary users," IET Communications, vol. 7, no. 17, pp. 1982-1992, 2013.

[21] R. Ahlswede, N. Cai, S.-Y. Li, and R. Yeung, "Network information flow," IEEE Transactions on Information Theory, vol. 46, no. 4, pp. 1204-1216, 2000.

[22] R. Yeung, "Network coding: A historical perspective," Proceedings of the IEEE, vol. 99, no. 3, pp. 366-371, 2011.

[23] S.-Y. Li, Q. Sun, and Z. Shao, "Linear network coding: Theory and algorithms," Proceedings of the IEEE, vol. 99, no. 3, pp. 372-387, 2011. 
[24] X. Zhang, A. Ghrayeb, and M. Hasna, "On relay assignment in networkcoded cooperative systems," IEEE Transactions on Wireless Communications, vol. 10, pp. 868 -876, March 2011.

[25] X. Li, T. Jiang, S. Cui, J. An, and Q. Zhang, "Cooperative communications based on rateless network coding in distributed mimo systems [coordinated and distributed mimo]," IEEE Wireless Communications,, vol. 17, pp. $60-67$, June 2010.

[26] S. Sharma, Y. Shi, J. Liu, Y. Hou, S. Kompella, and S. Midkiff, "Network Coding in Cooperative Communications: Friend or Foe?", IEEE Transactions on Mobile Computing, vol. 11, no. 7, pp. 1073-1085, 2012.

[27] L. Li, L. Wang, and L. Hanzo, "Generalized adaptive network coding aided successive relaying for noncoherent cooperation," IEEE Transactions on Communications, vol. 61, pp. 1750-1763, May 2013.

[28] S. Wang, Y. Sagduyu, J. Zhang, and J. H. Li, "Spectrum shaping via network coding in cognitive radio networks," in 2011 Proceedings IEEE INFOCOM, pp. 396-400, 2011.

[29] L. Hanzo, T. H. Liew, B. L. Yeap, R. Y. S. Tee, and S. X. Ng, Turbo Coding, Turbo Equalisation and Space-Time Coding(EXIT-Chart-Aided Near-Capacity Designs for Wireless Channels). John wiley-IEEE Press, 2011.

[30] S. X. Ng, O. Alamri, Y. Li, J. Kliewer and L. Hanzo, "Near-capacity turbo trellis coded modulation design based on EXIT charts and union bounds," IEEE Transactions on Communications, vol. 56, pp. 20302039, December 2008.

[31] L. Kong, S. X. Ng, R. Maunder, and L. Hanzo, "Maximum-throughput irregular distributed space-time code for near-capacity cooperative communications," IEEE Transactions on Vehicular Technology, vol. 59, pp. $1511-1517$, March 2010.

[32] H. Ochiai, P. Mitran, and V. Tarokh, "Design and analysis of collaborative diversity protocols for wireless sensor networks," in IEEE 60th, Vehicular Technology Conference, 2004. VTC2004-Fall. 2004, vol. 7, pp. $4645-4649$ Vol. 7, Sept. 2004.

[33] A. Tobagi Fouad and M. M. Hira, "Joint optimization of physical layer parameters and routing in wireless mesh networks," in The 9th IFIP Annual Mediterranean, Ad Hoc Networking Workshop (Med-Hoc-Net), 2010 , pp. $1-8$, June 2010.

[34] L. Hanzo, S. X. Ng, T. Keller and W. Webb, Quadrature Amplitude Modulation:From Basics to Adaptive Trellis-Coded, Turbo-Equalised and Space-Time Coded OFDM, CDMA and MC-CDMA Systems. WileyIEEE Press, 2004.

[35] G. Ungerböck, "Channel coding with multilevel/phase signals," IEEE Transactions on Information Theory, vol. IT-28, pp. 55-67, January 1982.

[36] SAGE, Open source mathematics software. Available: http://wwwsagemath.org/.

[37] C. W. L. Hanzo and M. Yee, Adaptive Wireless Transceivers: TurboCoded, Turbo-Equalized and Space-Time Coded TDMA, CDMA and OFDM Systems. John Wiley Sons, Ltd, 2002.

[38] L. Hanzo, L. L. Yang, E. L. Kuan and K. Yen, Single- and Multi-Carrier DS-CDMA: Multi-User Detection, Space-Time Spreading, Synchronisation, Networking and Standards. IEEE Wiley Press, 2003.

[39] H. V. Nguyen, Z. barbar, S. X. Ng, M. Mazzotti, L. Iacobelli and L. Hanzo, "Network coded MIMO aided cooperative communications in the ambulance-and-emergency area," in MoWNet'2014, 2014.

[40] H. V. Nguyen, S. X. Ng, and L. Hanzo, "Irregular convolution and unityrate coded network-coding for cooperative multi-user communications," IEEE Transactions on Wireless Communications, vol. 12, pp. 12311243, March 2013.

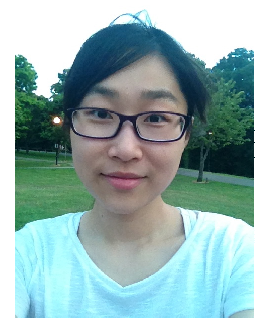

Wei Liang received her M.Sc. degree in wireless communication from University of Southampton, Southampton, U.K in 2010. She is currently working toward the Ph.D. degree in the Southampton Wireless Group at University of Southampton, Southampton, U.K. Her current research interests include adaptive coded modulation, netwrok coding, cooperative communication and cognitive radio network.

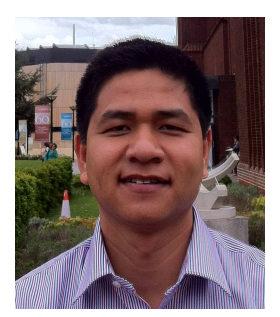

Hung Viet Nguyen received the B.Eng. degree in Electronics and Telecommunications from Hanoi University of Science and Technology (HUST), Hanoi, Vietnam, in 1999, the M.Eng. in Telecommunications from Asian Institute of Technology (AIT), Bangkok, Thailand, in 2002 and the Ph.D. degree in wireless communications from the University of Southampton, Southampton, U.K., in 2013. Since 1999 he has been a lecturer at the Post and Telecommunications Institute of Technology (PTIT), Vietnam. He is now a postdoc at Southampton Wireless (SW) group, University of Southampton. His research interests include cooperative communications, channel coding, network coding and quantum communications.

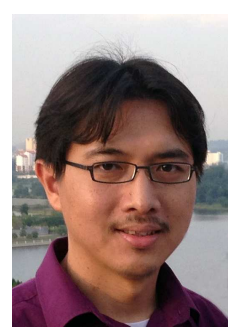

Dr Soon Xin Ng (S'99-M'03-SM'08) received the B.Eng. degree (First class) in electronic engineering and the Ph.D. degree in telecommunications from the University of Southampton, Southampton, U.K., in 1999 and 2002, respectively. From 2003 to 2006, he was a postdoctoral research fellow working on collaborative European research projects known as SCOUT, NEWCOM and PHOENIX. Since August 2006, he has been a member of academic staff in the School of Electronics and Computer Science, University of Southampton. He is involved in the OPTIMIX and CONCERTO European projects as well as the IU-ATC and UC4G projects. He is currently an Associate Professor in telecommunications at the University of Southampton.

His research interests include adaptive coded modulation, coded modulation, channel coding, space-time coding, joint source and channel coding, iterative detection, OFDM, MIMO, cooperative communications, distributed coding, quantum error correction codes and joint wireless-and-optical-fibre communications. He has published over 180 papers and co-authored two John Wiley/IEEE Press books in this field. He is a Senior Member of the IEEE, a Chartered Engineer and a Fellow of the Higher Education Academy in the UK.

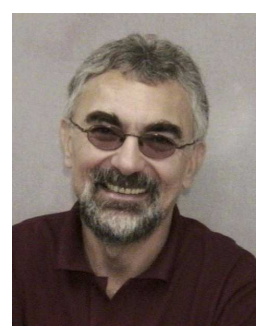

Lajos Hanzo (http://www-mobile.ecs.soton.ac.uk) FREng, FIEEE, FIET, Fellow of EURASIP, DSc received his degree in electronics in 1976 and his doctorate in 1983. In 2009 he was awarded the honorary doctorate "Doctor Honoris Causa" by the Technical University of Budapest. During his 37 -year career in telecommunications he has held various research and academic posts in Hungary, Germany and the UK. Since 1986 he has been with the School of Electronics and Computer Science, University of Southampton, UK, where he holds the chair in telecommunications. He has successfully supervised $80+\mathrm{PhD}$ students, co-authored 20 John Wiley/IEEE Press books on mobile radio communications totalling in excess of 10000 pages, published $1400+$ research entries at IEEE Xplore, acted both as TPC and General Chair of IEEE conferences, presented keynote lectures and has been awarded a number of distinctions. Currently he is directing a 100-strong academic research team, working on a range of research projects in the field of wireless multimedia communications sponsored by industry, the Engineering and Physical Sciences Research Council (EPSRC) UK, the European Research Council's Advanced Fellow Grant and the Royal Society's Wolfson Research Merit Award. He is an enthusiastic supporter of industrial and academic liaison and he offers a range of industrial courses. He is also a Governor of the IEEE VTS. During 2008 - 2012 he was the Editor-in-Chief of the IEEE Press and a Chaired Professor also at Tsinghua University, Beijing. His research is funded by the European Research Council's Senior Research Fellow Grant. For further information on research in progress and associated publications please refer to http://www-mobile.ecs.soton.ac.uk Lajos has 19 000+ citations. 